condition, whether or not they have symptoms of UTI. The development of renal scarring in children with VUR may then be prevented using prophylactic antibiotic treatment or early ureteric reimplantation.

Original article Ataei $\mathrm{N}$ et al. (2004) Screening for vesicoureteral reflux and renal scars in siblings of children with known reflux. Pediatr Nephrol 19: 1127-1131

\section{End-fire ultrasound probes in prostate cancer diagnosis}

Transrectal ultrasound-guided biopsy is a widely accepted procedure in prostate cancer diagnosis, although little is known about the effects on the detection rate of different types of ultrasound equipment. Paul et al. hypothesized that end-fire ultrasound probes, which facilitate sampling in the most lateral part of the peripheral zone, would be superior to side-fire probes in the detection of smaller tumors. Their retrospective study comparing these two types of probe has recently been published.

A total of 2,625 patients underwent a firsttime, systematic sextant biopsy using the Kretz Combisone side-fire probe, the Bruel \& Kjaer Medical side-fire probe or the ATL HDI end-fire probe. The side-fire probes limited the prostate biopsy to a sagittal axis, whereas the end-fire probe allowed sampling in any section.

The overall prostate cancer detection rate (35.2\%) was similar using all three probes. In a subgroup of patients with a PSA level of $4-10 \mathrm{ng} / \mathrm{ml}$, however, the detection rate using the end-fire probe (31.3\%) was statistically significantly higher than with either of the side-fire probes $(P=0.01)$. This was also the case in a further subgroup of patients with nonpalpable cancer.

In summary, the end-fire probe provided a higher prostate cancer detection rate in two patient subgroups, compared with the sidefire probes. The authors suggest that this was due to improved visualization of the lateral peripheral zone, in which most peripheral zone tumors occur.

Original article Paul R et al. (2004) Influence of transrectal ultrasound probe on prostate cancer detection in transrectal ultrasound-guided sextant biopsy of prostate. Urology 64: 532-536

\section{Measuring urinary tract stones by computed tomography}

The determination of urinary tract stone size is an important step in planning appropriate treatment. Although the introduction of computed tomography (CT) has improved the accuracy of such measurements, standard axial CT images may not allow precise estimations in the craniocaudal plane. Nadler et al. have investigated the use of coronal imaging as an additional approach.

The team reviewed CT images from 102 patients (151 stones) who had undergone routine abdominal imaging over a 9-month period. Axial images were used to measure the length and width of each stone. Measurement of craniocaudal length and width was then carried out using reconstructed, contiguous coronal images. Finally, the total area of each stone was calculated using both the axial and coronal images.

Significant differences were found between the axial and coronal measurements of stone size. The mean greatest stone dimension on axial imaging was $4.87 \mathrm{~mm}$, compared with $6.51 \mathrm{~mm}$ on coronal imaging $(P<0.0001)$. Axial imaging also underestimated the mean stone area and overestimated craniocaudal length by comparison with the coronal measurements.

Concluding that axial imaging does not allow accurate measurement of stone dimensions, the authors recommend the addition of routine coronal imaging in this setting.

Original article Nadler RB et al. (2004) Coronal imaging to assess urinary tract stone size. J Urol 172: 962-964

\section{Advanced refractory prostate cancer: new treatment trial}

Men with metastatic, androgen-independent prostate cancer have a median survival of 1 year or less. Current treatment with mito-xantrone plus prednisone or hydrocortisone palliates bone pain in some patients, but no available therapies prolong survival. Phase I and II studies have shown improved survival in patients receiving docetaxel plus estramustine; Petrylak and colleagues have investigated this in a randomized, phase III trial.

A total of 770 men with metastatic, hormoneindependent prostate cancer were prospectively enrolled in the study. Of 674 eligible patients, half were assigned to receive docetaxel plus 